\title{
A TESTBED FOR HIGH VOLTAGE, HIGH BANDWIDTH CHARACTERIZATION OF NONLINEAR DIELECTRICS
}

\author{
F.J. Zutavern ${ }^{\xi}$, G.L. Brennecka, S.F. Glover, \\ G.E. Pena, G.J. Denison, J.M. Rudys \\ "Sandia National Laboratories, Albuquerque, NM 87185 USA
}

\begin{abstract}
The dielectric response of many high permittivity materials is nonlinear with both field and frequency. For example, ferroelectric materials exhibit a hysteretic polarization - electric field (P-E) response similar to the B-H curve of ferromagnetic materials. These P-E hysteresis loops are typically measured at low frequencies; the material behavior at high frequencies is less understood. To address this information gap, a test bed has been created to characterize non-linear material behavior at high frequencies and high voltages. This paper will report testbed goals in addition to design, assembly, analysis, and issues. Preliminary results will also be presented from commercially available nonlinear capacitors and in-house fabricated ferroelectric materials, including $10 \mathrm{nF}$ non-linear $\mathrm{BaTiO}_{3}$-based capacitors and 3 $\mathrm{mm}$ thick lead zirconate titanate (PZT)-based materials.
\end{abstract}

\section{INTRODUCTION}

All high permittivity materials exhibit some degree of nonlinear dielectric (NLD) response with applied electric field, and for most, this involves saturation (decrease) of the small-signal permittivity under increasing DC field. A particularly interesting and challenging case is that of ferroelectric (FE) materials. Their large permittivities make FEs the basis for the ceramic capacitor industry, and field-switchable spontaneous polarization underlies their use for nonvolatile memory (FERAM), piezoelectric, and many other applications. A complete representation of the dielectric response of such materials requires a full description of both monotonic field saturation and frequency dispersion of relevant polarization mechanisms as well as the dynamic response of the polarization reversal associated with ferroelectric switching.

Accurate description of the NLD response of high permittivity (FE) materials at high fields and high frequencies requires direct measurement. The signature PE hysteresis loop of ferroelectrics, however, is generally measured under low-current conditions. Existing work on the exposure and response of FEs to brief high-field pulses has focused on thin films under large electric fields but low voltages [1-3], and it has been well established that the differences in chemical, interface, and strain conditions contribute to significant differences in the observed response(s) between thin films and bulk ceramics. In order to help fill the significant knowledge gap associated with the dynamic response of NLD, this paper describes the design and construction of a custom test bed for accurate measurement of this nonlinear response of high permittivity bulk ceramics in the large electric field and high frequency regimes.

\section{EXPERIMENTAL DESIGN}

The main goal of the NLD test bed is to drive a $1-50 \mathrm{nF}$ FE sample around its P-E loop with a low inductance circuit $(10 \mathrm{nH})$ and high bandwidth $(1 \mathrm{GHz})$ diagnostics at voltages up to $20 \mathrm{kV}$ and currents up to $10 \mathrm{kA}$. A secondary goal is to drive the sample through P-E subloops to measure the sample response at high frequencies within the P-E loop. Polarization of the sample is obtained by integrating the current passing through the sample and dividing by its cross-sectional area. The current is measured with a $0.0505 \Omega, 1.2 \mathrm{GHz}$ bandwidth current viewing resistor (T\&M A-1-05). The electric field is determined by measuring the voltage across the sample and dividing by its thickness. Calibration of the voltage is determined with a $80 \mathrm{MHz}, 40 \mathrm{kV}$, compensated, high impedance, resistive probe (North Star PVM-1 1000:1 HV). A $\sim 5 \mathrm{GHz}$ V-dot probe (SNL Dennison) is used to record the high bandwidth voltage waveform. This is automatically calibrated by fitting it to the resistive probe waveform in two low-bandwidth early and late-time intervals: (1) the pre-switching baseline and (2) postswitching polarization saturation. Simulation of the circuit and analysis of the data require modeling of the sample behavior internal to the P-E loop and along its boundaries. A hyperbolic tangent that depends on the initial values of $\mathrm{P}$ and $\mathrm{E}$ and the polarity of the current provides a reasonable model for low bandwidth measurements of most materials. However P-E loops with sharper corners and/or a strong variation with frequency require a model with a higher order exponential dependence, and a free parameter to characterize the sharpness of the corners independent of the slope of the loop through zero polarization.

The circuit for the NLD testbed has gone through several iterations. The circuit requirements are to drive a relatively large capacitance from a charged high voltage condition rapidly to a similar voltage of the opposite polarity. A capacitive discharge circuit was chosen to supply the required current to drive the sample rapidly through a polarity reversal to polarization saturation. In the first configuration, a pair of high voltage relays were used to reverse the connections on the sample after the current pulse decays to zero and the switch opens. In this

\footnotetext{
* Sandia National Laboratories is a multi-program laboratory managed and operated by Sandia Corporation, a wholly owned subsidiary of Lockheed Martin Corporation, for the U.S. DOE's National Nuclear Security Admin. under contract DE-AC04-94AL85000, SAND \# 2013-5245C. .This project supported in part by the Laboratory Directed Research and Development program at SNL. U.S. Government work not protected by U.S. copyright 
way, one HV power supply and one single-polarity, wellconditioned switch could be used to drive the sample around the full P-E loop. The disadvantages of the relays were their added inductance, which reduced the effective bandwidth of the circuit, and their requirement for a timing circuit to control the relays, trigger the low-jitter HV switch, and allow for relay switching and settling time. Since P-E loops are symmetric for most materials and only the "sides" of the loop need to be measured rapidly, only one side of the loop needs to be pulsed to measure the loop. A single relay and HV power supply can be used to drive the sample into saturation for reproducible initial conditions. Then a low-jitter, HV, well-conditioned switch and another HV supply of the opposite polarity can be used to drive the sample through one leg of the P-E loop. The symmetry of the P-E loop can be checked by manually changing the connections to the sample and repeating the test procedure.

Another circuit change was the location of the HV lowjtter switch, a triggered spark gap (TSG) (EG\&G GP20A, Perkin Elmer GP-20B). Initially, the recommended configuration of this switch was selected, where the cathode is connected to ground. Preliminary testing with other switch locations had shown significant trigger current passing through the FE sample. By grounding the cathode, the trigger current returns directly to the trigger pulser without passing through the load (FE sample). However, this placement of the switch puts a DC bias on the voltage monitors, the initial charge voltage on the sample relative to ground, or equivalently the voltage across the switch before it is triggered. With the switch grounded, the monitors are measuring the voltage across the switch and the sample. This forces the voltage monitors to change voltage suddenly when the switch closes, before charge is transferred. The low BW monitor cannot follow this ideally infinite risetime voltage step, so the intial baseline interval is useless for voltage calibration of the V-dot. Furthermore, a fast edge excites oscillations in the small stray inductances of the monitor connections to the circuit, which can be seen by the high BW V-dot and are not representative of the voltage across the FE sample, which changes relatively slowly as it

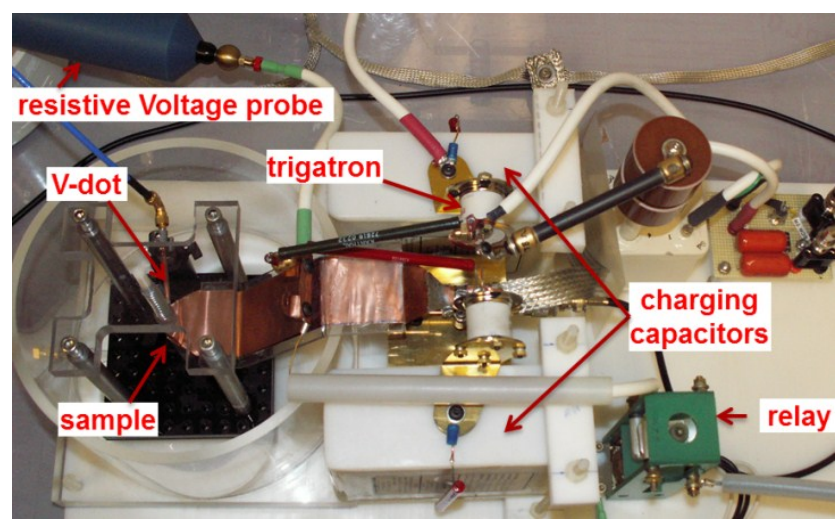

Figure 1. The NLD testbed is shown in this picture.

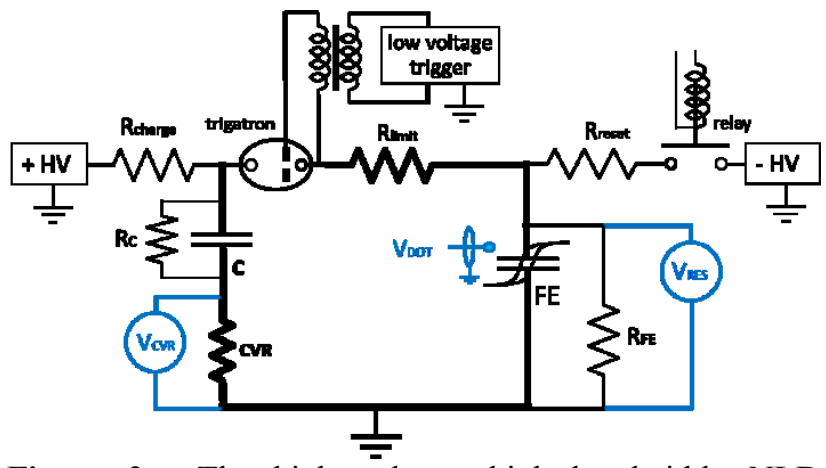

Figure 2. The high voltage, high bandwidth, NLD testbed is shown above. Component values are in Table I.

accumulates charge. Differential monitor pairs cannot eliminate these issues, because they would both be measuring this high voltage step, and subtracting two HV high BW measurements will produce more noise than the relative small initial change in voltage that is developing across the FE sample. To eliminate this extraneous voltage step on the monitors, which measure voltage relative to ground, one side of the FE sample must be grounded, and the switch must be "floating" at high voltage. The switch trigger current, introduced in the FE sample by floating the switch, can be significanlty reduced by floating the high voltage trigger pulse with a HV trigger transformer with low capacitive coupling between the primary and the secondary coils. Considering the high current anticipated in these tests, the switch trigger current is probably a smaller source of error than the sudden voltage step on the monitors.

\section{EXPERIMENTAL SETUP}

A picture of the test bed present configuration is shown in figure 1. Two TSGs are in this picture for a future tests. Only the top TSG was being used in these tests. The latest circuit used for these measurements is in Figure 2 with component values in Table I. A relay is used to charge the FE sample to its initial voltage before a high current TSG drives the sample to the opposite polarity. A low voltage pulse generator is used to control the relay and TSG timing, so system operation is reproducible and repetitive. If the FE sample remains stable during the test pulse, many shots are acquired to assess the reproducibility of the results.

Table I. Component values for the circuit in Figure 2.

\begin{tabular}{c|c|c|c|c|c|c}
$\mathrm{R}_{\text {charge }}$ & $\mathrm{R}_{\text {limit }}$ & $\mathrm{R}_{\text {reset }}$ & $\mathrm{R}_{\mathrm{FE}}$ & $\mathrm{R}_{\mathrm{C}}$ & $\mathrm{R}_{\mathrm{CVR}}$ & $\mathrm{C}$ \\
\hline 50 & $10-1000$ & 100 & 1 & 100 & 50.5 & 75 \\
$\mathrm{k} \Omega$ & $\Omega$ & $\mathrm{k} \Omega$ & $\mathrm{G} \Omega$ & $\mathrm{M} \Omega$ & $\mathrm{m} \Omega$ & $\mathrm{nF}$
\end{tabular}

\section{RESULTS}

Measurements of current, voltage, and polarization versus electric field (P-E loops) are shown in figures 3 thru 8 for $\mathrm{BaTiO}_{3}$, PLZT, and $\mathrm{LiTaO}_{3}$ with current limited by 1000 and $100 \Omega$ resistors. 

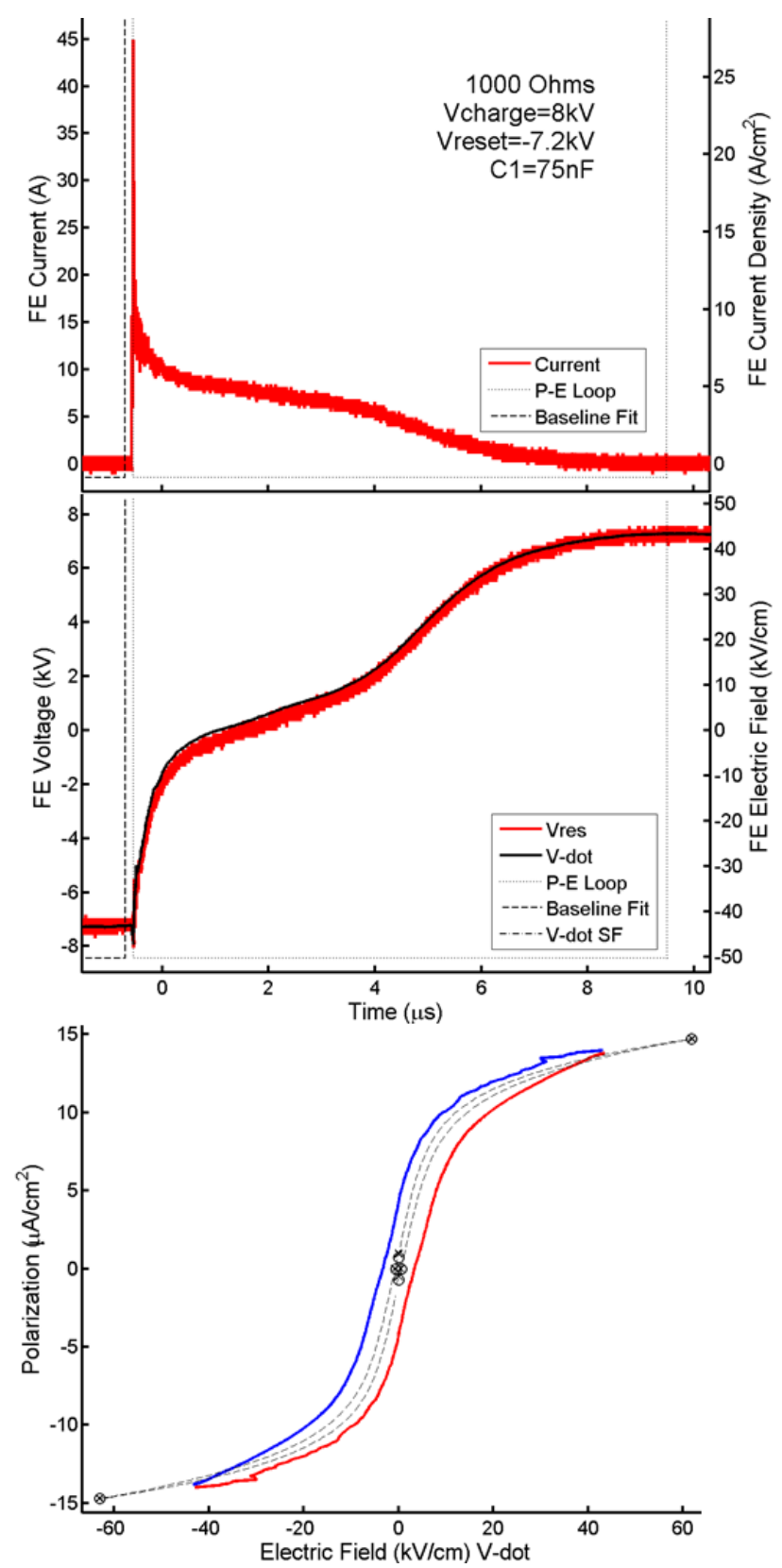

Figure 3. Results for non-linear $\mathrm{BaTiO}_{3}$ capacitors are shown with current limited by $1 \mathrm{k} \Omega$ : (top) current and current density, (middle) voltage and electric field, (bottom) high BW P-E loop based on V-dot monitor and CVR (red curve) and low BW P-E loop (black dashed). The upper left leg of the P-E loop (blue) is the symmetric reflection of the lower right leg (red). The dashed curve is the low bandwidth ( DC) P-E loop [4].

Figure 3 shows that the relatively narrow P-E loop of $\mathrm{BaTiO}_{3}$ is reproduced in the 10 microsecond regime, although it is somewhat wider and approaches a slightly higher polarization saturation with increasing electric field.
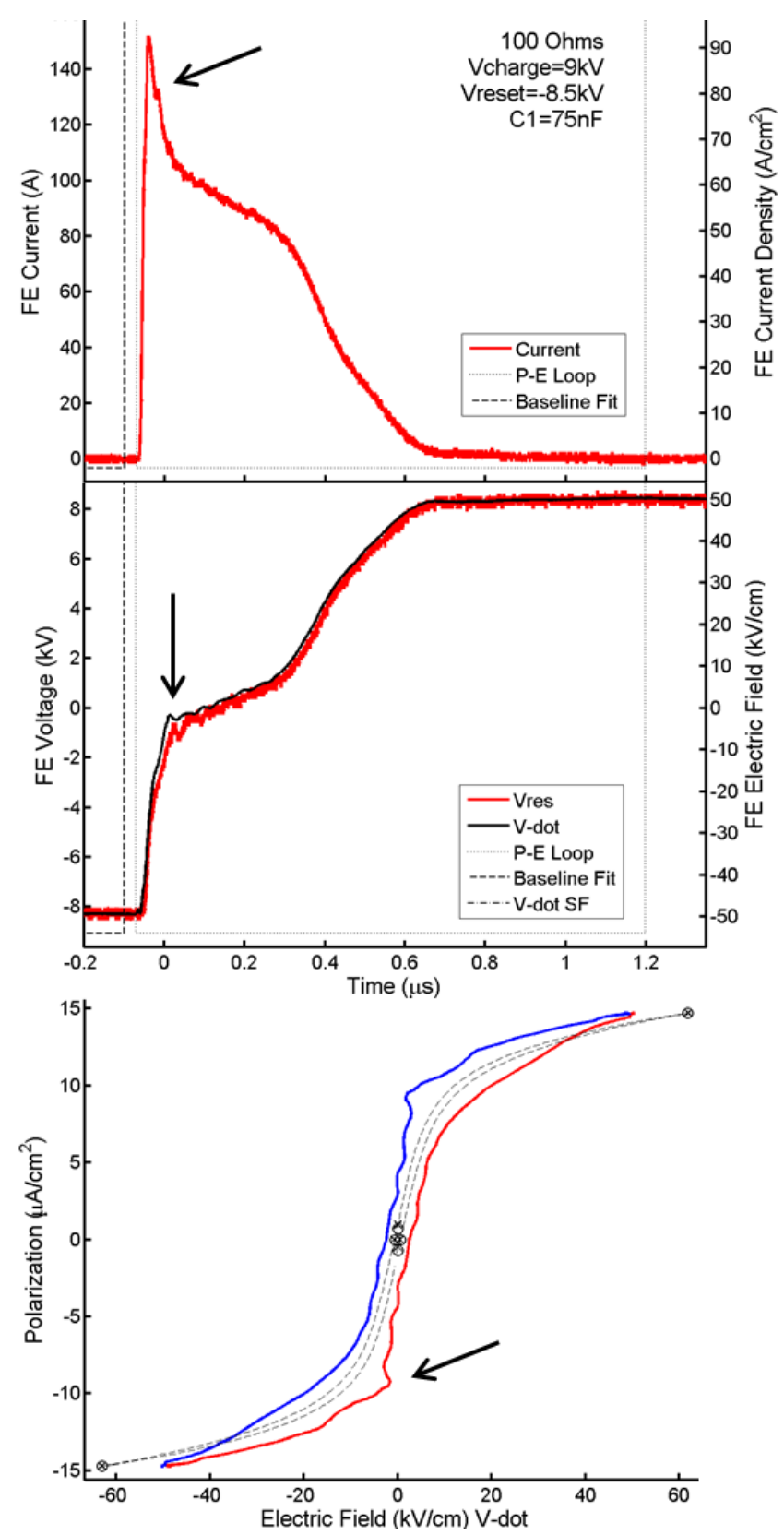

Figure 4. Results for non-linear $\mathrm{BaTiO}_{3}$ capacitors are shown with the current limited by $100 \Omega$. A faster increase in electric field across the sample and a distortion at the knee of the P-E curve appear in these plots (arrows). The blue curve, the symmetric reflection of the red curve, emphasizes the issues in the P-E loop width that are caused by this distortion. The dashed curve is the low bandwidth ( DC) P-E loop [4].

The faster curves in figure 4 show some "disturbance" near the initial peaks in current and voltage resulting in a sharper "knee" in the P-E loop (arrows). The initial larger polarization is chosen for vertically symmetry about zero polarization, which influences the loop width. 

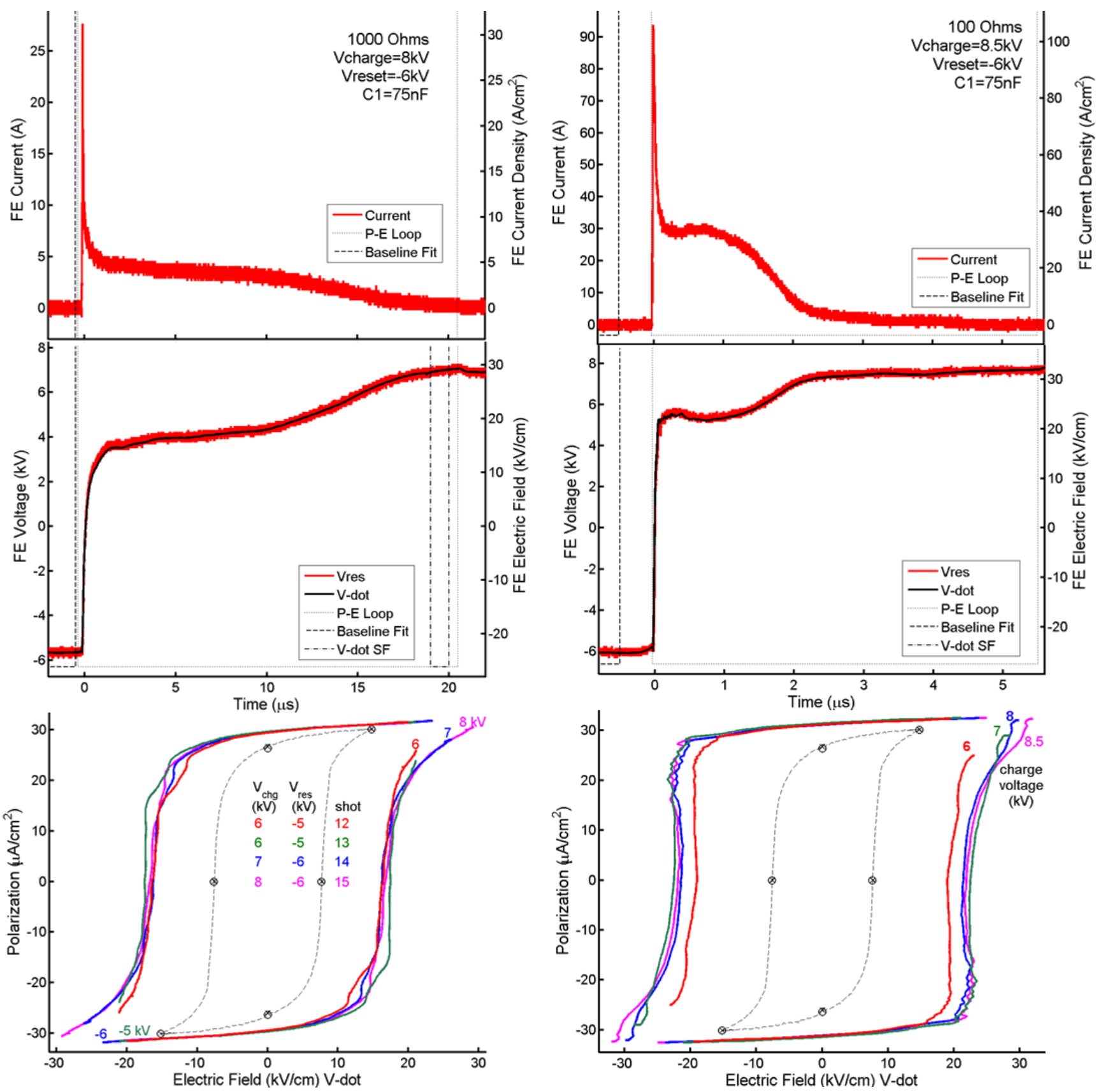

Figure 5. These measurements were obtained from PLZT with the current limited by $1 \mathrm{k} \Omega$. The bottom plot displays the P-E curves from 4 shots (12-15), whereas the current (top) and voltage (middle) are displayed only for shot 15. The upper-left legs of the P-E loops are the symmetric reflections of the lower right legs. The dashed curve is the low bandwidth ( DC) P-E loop. [4]

PLZT has a much wider P-E loop than $\mathrm{BaTiO}_{3}$ at very low frequencies (dashed). Its loop widens even more in the $20 \mu$ s (figure 5) and $5 \mu$ s (figure 6) regimes. The lower right knee of the P-E loops also show some shot-toshot variation which appears to exaggerate the variation in the loop width.

Figure 6. PLZT measurements when the current was limited with $100 \Omega$ are shown above. Faster charging produces sharper corners and noisier knees. P-E loops are shown for 4 shots, whereas the current (top) and voltage (middle) are displayed only for the shot charged to $8.5 \mathrm{kV}$. All shots had the same reset voltage $(-6 \mathrm{kV})$. The dashed curve is the low bandwidth ( DC) P-E loop [4].

The heights of these loops, based on vertical symmetry about zero polarization is more difficult to determine with this data which does not start with enough reset voltage to "close" the loops and also shows some shot-to-shot noise in the voltage near the saturation polarization. 

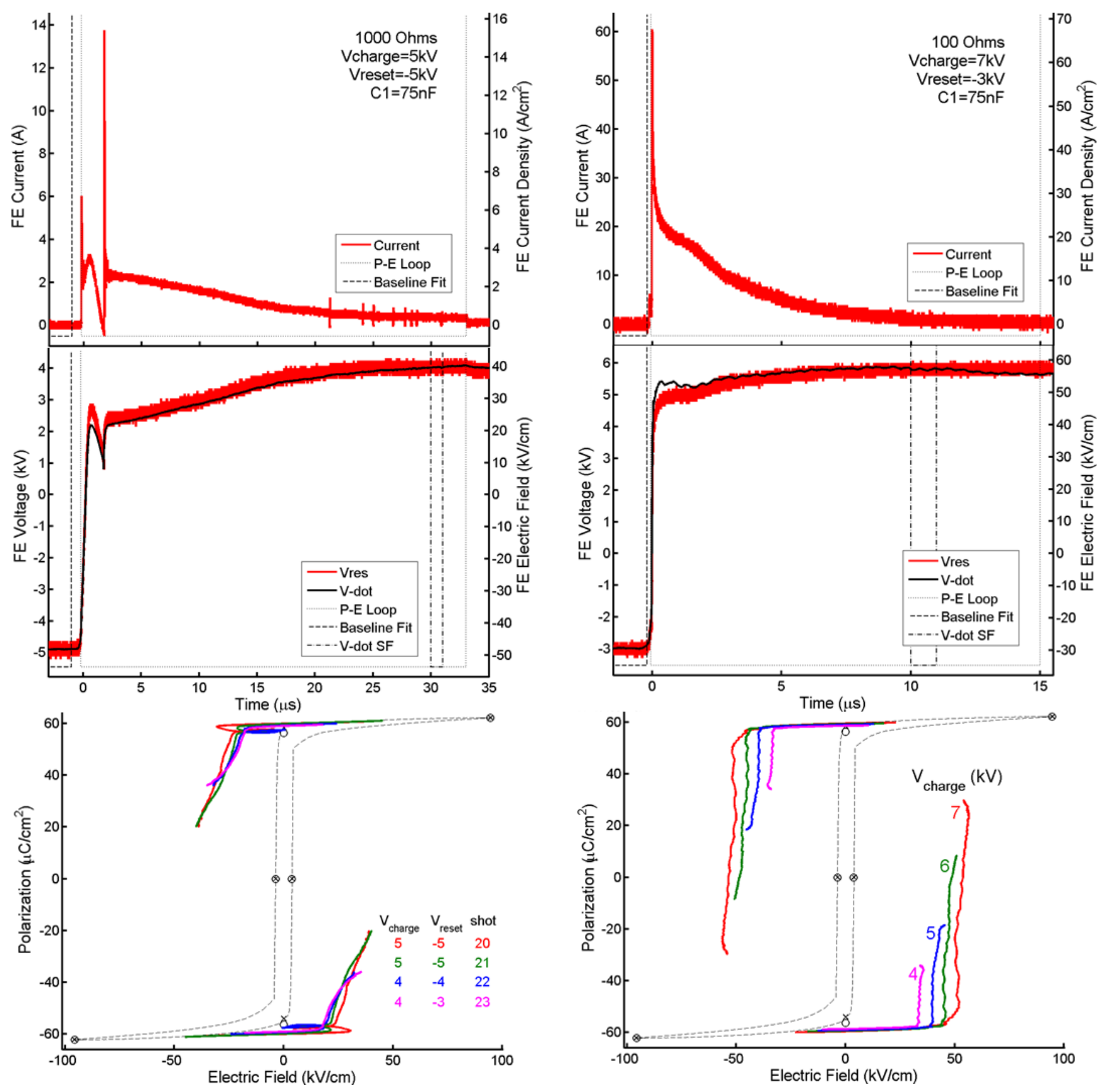

Figure 7. These measurements from $\mathrm{LiTaO}_{3}$ had the current limited by $1 \mathrm{k} \Omega$. The bottom plot displays the PE curves from 4 shots (20-23). The current (top) and voltage (middle) are displayed only for shot 21 . The upper-left legs of the P-E loops are the symmetric reflections of the lower right legs. The dashed curve is the low bandwidth ( DC) P-E loop. [4]

$\mathrm{LiTaO}_{3}$ samples did not survive the fields required to measure complete P-E loops. In addition to electrical stress, the rapid increase in electric field produces large mechanical stress due to their piezoelectric properties. The samples appeared to reach higher fields in the $10 \mu \mathrm{s}$ regime, but only a small number of samples were tested.

Figure 8. $\mathrm{LiTaO}_{3}$ curves are plotted with the current limited by $100 \Omega$. Faster charging produces nearly right angle corners and smoother knees. P-E loops are shown for 4 shots, whereas the current (top) and voltage (middle) are displayed only for the shot charged to \& $7 \mathrm{kV}$. In this case, all shots had the same reset voltage $(-6 \mathrm{kV})$. The dashed curve is the low bandwidth ( DC) P-E loop. [4]

As with the PLZT samples, the faster pulses produce sharper corners and wider loops. The $\mathrm{LiTaO}_{3}$ samples also show an apparent increase in width with increasing charge voltage which may have also been observe with the PLZT if they had been tested with lower charge voltages. Apparently there is a delay in the response of 
the ferroelectric domains when the electric field "releases" them from saturation rapidly.

Many of these curves show "disturbance" or noise near the "knees" of the curves where the differential capacitance is decreasing rapidly. The sudden reduction in current and leveling-off or even decrease in voltage across the sample may be producing a sudden loss of voltage across the switch due to circuit inductance. These conditions may cause the TSG resistance to increase as carriers recombine faster than they are being generated by the reduced field. If the switch is attempting to open, its oscillatory behavior would distort the P-E loop as is observed. Reduced inductance, improved switch triggering, or a more stable switch with higher currents or longer recombination times might reduce this effect.

The time scales for the current and voltage curves in the preceding figures were all set to cover the time range used for plotting the P-E loops (light gray dotted lines). This range depended not only on the current limiting resistors

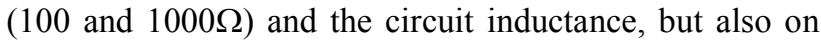
the non-linear capacitance of the samples, with wider P-E loops requiring more time to charge. The narrow P-E loops for the $\mathrm{BaTiO}_{3}$ capacitors charged nearly nine times faster with the $100 \Omega$ limiting resistor. However, the wider loops for PLZT and $\mathrm{LiTaO}_{3}$ samples (which widened even more with the fastest pulses) only charged four and two times faster, respectively, with $100 \Omega$.

The initial values for the sample polarization in these measurements are determined by assuming that the sample was reset to the lower boundary (polarization saturation) before the TSG was switched. In cases where the sample was driven close to saturation near the upper right boundary, vertical symmetry of the loop about zero polarization could be used to determine the initial polarization. However, when samples were not driven close to saturation, the "DC" value of the polarization at the reset field was used as the initial polarization of the higher BW loops. This symmetry was observed not only in the "DC" loops, but also in previous tests, where high voltage relays were used to rapidly change the polarity of the sample between the fast capacitive discharge pulses. Future tests are planned using two fast discharge switches and dual polarity power supplies to drive the samples around all sides of the P-E loops. Charge voltages will also be adjusted to measure sets P-E traces internal to the P-E loops.

\section{V.CONCLUSION}

In this paper, we have described the assembly and testing of a high voltage, high bandwidth test bed for materials with non-linear dielectric polarizability. The choices made for the selected configuration and circuit components were discussed, and issues with initial configurations were explained. Three examples of ferroelectric components $\left(\mathrm{BaTiO}_{3}, \mathrm{PLZT}, \mathrm{LiTaO}_{3}\right)$ were tested at in two different time regimes (10-30 and 1-15 $\mu \mathrm{s})$ at $4-8 \mathrm{kV}$ or $30-60 \mathrm{kV} / \mathrm{cm}$. The non-linear polarization versus electric field was plotted and shown to be remarkably different than the same measurements made at very low frequencies ("DC"). In general, P-E loops widened, corners sharpened, and saturation values increased as the rate of change in the electric field increased.

\section{ACKNOWLEDGEMENTS}

The authors would like to acknowledge the extensive work by the late Kim Reed to explore and develop nonlinear ferroelectric materials for pulsed power applications. These efforts led to the realization that the material properties of these materials should be measured in large samples at high fields and high frequencies.

\section{REFERENCES}

[1] J. Li, B. Nagaraj, H. Liang, W. Cao, Chi. H. Lee, and R. Ramesh, "Ultrafast polarization switching in thin-film ferroelectrics," Appl. Phys. Lett., 84[7] 1174-6 (2004).

[2] A. Grigoriev, D.-H. Do, D.M. Kim, C.-B. Eom, B. Adams, E.M. Dufresne, and P.G. Evans, "Nanosecond Domain Wall Dynamics in Ferroelectric $\mathrm{Pb}(\mathrm{Zr}, \mathrm{Ti}) \mathrm{O}_{3}$ Thin Films," Phys. Rev. Lett., 96, 187601 (2006).

[3] I.K. Bdikin, A.L. Kholkin, A.N. Morozovska, S.V. Svechnikov, S. -H. Kim, and S.V. Kalinin, "Domain dynamics in piezoresponse force spectroscopy: Quantitative deconvolution and hysteresis loop fine structure," Appl. Phys. Lett., 92182909 (2008).

[4] Low frequency $(0.03-1.0 \mathrm{~Hz}) \mathrm{P}-\mathrm{E}$ data were collected using a Precision Workstation ferroelectric test system from Radiant Technologies (Albuquerque, NM). 